\title{
Trust is Good, Control is Better - Customer Preferences Regarding Control in Teleoperated and Autonomous Vehicles
}

\author{
Katharina Keller \\ Goethe University Frankfurt \\ kakeller@,wiwi.uni-frankfurt.de \\ Jan Zibuschka \\ Robert Bosch GmbH \\ jan.zibuschka@de.bosch.com
}

\author{
Christian Zimmermann \\ Robert Bosch GmbH \\ christian.zimmermann3@de.bosch.com \\ Oliver Hinz \\ Goethe University Frankfurt \\ hinz@wiwi.uni-frankfurt.de
}

\begin{abstract}
Autonomous vehicles are continually being developed and are the focus of many research projects. While the focus so far has been primarily on technical features and the implementation of autonomous vehicles, we are turning to the consumer side to analyze the preferences of potential users regarding teleoperable robotaxis. After all, user acceptance is a necessary condition for the success of a new technology. Using a choice-based conjoint analysis, we investigate preferences for three different control dependent attributes and the price. We observe price to be the most important attribute, followed by the possibility of intervention, pilot, and interior monitoring - in this order. However, respondents' preferences turn out to be very heterogeneous. Within the framework of a cluster analysis, we look at the results in segments and analyze possible moderating effects using an analysis of variance.
\end{abstract}

\section{Introduction}

Autonomous vehicles (AVs) are envisioned to increase traffic safety, efficiency, and carry the promise to unburden drivers from driving tasks, effectively making them passengers. Prototypes of SAE level 4 and 5 vehicles (i.e., highly or fully automated vehicles, cf. [39]) are already tested on public roads. Many of these vehicles are utilized as socalled "robotaxis" that customers can order similarly to traditional taxis to pick them up and drop them off at customer-defined locations. Robotaxi prototypes are currently operated or have been operated by, e.g., Mercedes and Bosch or Waymo [14].

There are, however, consumer concerns about the use of AVs in general, for example, loss of control over the car [11]. Some users also lack confidence in the technology [6]. Besides, current AVs are pushed to their limits by everyday use under real, e.g., urban traffic conditions, although these highly automated or AVs are actively researched, developed, and tested. In this regard, teleoperation can aid as a measure for a smooth transition from non-autonomous to autonomous driving [18] by providing a fallback in certain situations, but it is also at the same time a potential enabler for future AV use cases.

Teleoperation of vehicles is a promising approach to alleviate the problems of robotaxis under challenging traffic conditions [18]. For example, a remote human operator can take control of the robotaxi if it is in a situation where it is unable to decide on its next action, e.g., due to insufficient sensor information or if it would need to violate traffic rules in order to continue driving. The latter could be the case, for instance, when a police officer has to reroute traffic after an accident and vehicles need to drive over a curb.

In this paper, we investigate customers' attitudes and preferences regarding teleoperable robotaxis and aspects of control for this application of AVs. We present the results of a choice-based conjoint analysis that elicits prospective consumers' preferences for important attributes that were identified in expert interviews beforehand. We use this information to determine different customer segments and their preferences for the design of autonomous taxis.

The paper is structured as follows: We provide a literature review of current research on autonomous vehicles, focusing on consumer preferences regarding (shared) autonomous mobility services and the factors trust, risk, and control. Subsequently, we outline our empirical study with its study setup, data collection, and model assessment. We then discuss the presented results and conclude the article with possible implications for theory and practice, limitations, and an outlook on future research. 


\section{Previous Research}

While the research field of AVs in general has developed into a considerable literature stream in recent years [13], most of this work is concerned with the technical properties of AVs and does not focus on robotaxis and teleoperation. Moreover, research contributions from the consumer perspective are relatively sparse [3], although consumer preferences indicate a high degree of heterogeneity in this area, warranting further research. We first provide an overview on consumer preferences regarding AVs in general and subsequently focus on trust, risk, and control aspects.

\subsection{Consumer Preferences Regarding AVs}

For example, Maeng and Cho [29] performed a choice-based conjoint analysis with South Korean consumers for networked AVs interacting with roadside infrastructure. They found heterogeneous preferences with high relevance of the infrastructural aspects. According to their findings, some consumers seem to envision exclusive infrastructure for AVs, while others reject the concept altogether. Similarly, Daziano et al. [7] found a significant additional willingness to pay for automation in vehicles, but also found that this varies greatly depending on the consumer segment. They do find, however, that all participants prefer full automation over partial automation or no automation. This is unsurprising given that automation levels requiring the passenger to be ready to take over control at any time are challenging for users as well as car manufacturers, some of which are considering to skip SAE Level 3 entirely [43]. The picture is further complicated by the various transport modalities subsumed under the umbrella of AVs. Papadima et al. [33] observed the ticket price as the most important attribute in the decision to opt for a driverless bus, with respondents not willing to pay more than for conventional bus service. Interestingly, their participants show no safety concerns regarding AVs and even prefer driverless buses over buses with specialized drivers or escorts. Hulse et al. [20] find that "autonomous cars were perceived as a 'somewhat low risk' form of transport" that were perceived "more risky when a passenger yet less risky when a pedestrian. Autonomous cars were also perceived as more risky than existing autonomous trains" [20]. Stoiber et al. [41] showed that there is a general acceptance of shared autonomous taxis. Abraham et al. [1] find that ride sharing is considered "an occasional alternative to driving" for a majority of respondents in their study.
As already discussed, personality traits and other individual psychological factors may also partially account for this heterogeneity. Nordhoff et al. [32] found that attitude towards technology, thrill-seeking behavior, and support of a car-free environment are essential predictors of AV acceptance.

Previous research also identifies various barriers to the adoption of self-driving vehicles. For example, consumers exhibiting high levels of driving enjoyment may perceive the move to AVs as undesirable [10]. However, it has been shown that several other factors prominent in the discussion on autonomous cars, such as location privacy or the advantage of being able to read, eat, or even sleep in the car, do not significantly influence adoption intentions [15].

\subsection{Trust, Risk, and Control}

The field of consumer trust, risk, and control perception is highly relevant to the adoption of AVs in general and robotaxis in particular but is similarly heterogeneous. Trust in the technical competence and situational awareness of the vehicle is an important a priori predictor for the adoption of AVs [6], with the transparency of the operation of the vehicle being a key factor for increasing trust. However, previous research shows different results. While Mühl et al. [30] show that passengers prefer and trust a human driver over an AV, Wintersberger and Riener [45] observe that front-seat passengers accept and trust AVs and human drivers equally. Moreover, Zoellick et al. [47] prove that passengers exhibit high values of acceptance, perceived safety, trust, and usage intention after a real experience in an autonomous driving bus.

Risk perception is broadly recognized as an essential driver, both regarding the safety of AVs [23] and other technological risk factors [15]. Individuals with high safety concerns related to current vehicles generally welcome self-driving technologies [10]. For users with more experience with AVs, the predictive power of risk perceptions decreases and classic adoption factors such as perceived usefulness become more important, while perceived ease of use is only relevant for experienced users [46]. Interestingly, cybersecurity risk perception does not seem to influence consumers' intention of adopting AVs [15]. How automation affects risk perception is not uniform:

While the attribute 'safety' has the highest relative importance [40], its interaction with the degree of automation depends on individual predispositions [40]. Overall, a high level of automation is perceived as most beneficial for safety [26], with trust and social presence as important mediating factors. Furthermore, the automation of vehicles leads to a feeling of loss of control [36], which drivers perceive as negative [28]. 
Howard and Dai [19] also find that "individuals were most concerned with liability, the cost of the technology, and losing control of the vehicle". A considerable segment of drivers shows an optimistic tendency regarding their control abilities in critical driving situations [8], suggesting that providing them with the ability to intervene improves the control over AVs perceived by the customer and thus reduces potential safety concerns, thereby increasing the acceptance of AVs.

\section{Empirical Study}

This paper aims to deepen the understanding of the above-described factors in consumers' acceptance of teleoperable robotaxis, using a mixed approach comprising qualitative results of an expert panel and a large-scale quantitative choice-based conjoint analysis [7], which estimates preferences for all attribute levels.

Survey-based choice-based conjoint analyses (CBCs) are a widely used econometric method to determine preferences or willingness to pay in an economic environment [15]. They have a solid foundation in sociology and behavioral research [34] and enable the analysis of choices between hypothetical goods. Respondents repeatedly select their most preferred alternative from a choice set of alternatives characterized by attributes and associated attribute levels. Based on these choices, the influence of the different attributes can be determined and, thus, valuable insights regarding the adoption behavior can be gained [7]. Participants, therefore, do not indicate their preferences directly, but these are derived from the selection decisions. Given the similarity of these decision situations to real purchase decisions, CBC analysis can explain actual purchasing behavior well, which has also been confirmed empirically [29]. We use Hierarchical Bayes for estimation, which is a powerful tool that provides the distributions of parameter estimates for respondents at the individual level with only 12 choices per respondent.

\subsection{Research Methodology and Study Setup}

In this section, we describe the selection of attributes and the corresponding attribute levels as well as the structure of our study which is displayed in Table 1. On the one hand, it is important to choose an appropriate number of attributes, as too few attributes can distort respondents' decisions by drawing their attention to some attributes on which they may not focus when the technology is actually implemented [27]. In addition, too many attributes result in lengthy and complicated surveys where respondents cannot answer precisely [27]. On the other hand, the attributes and their associated attribute levels must be chosen carefully, since the participants make their hypothetical decisions based on these attributes, which are the starting point for preference analysis. Especially for technologies that are not yet on the market, a rather small number of attributes should be chosen to avoid cognitive overload or confusion [34].

To identify attributes for the $\mathrm{CBC}$, we employed the Nominal Group Technique (NGT) [9] with a group of experts from both the automotive industry and a research institute, including group and department heads, chief and senior experts, and researchers from the AV domain. The NGT can be used to generate and rank ideas in a group setting while avoiding "domination by certain group members" [37].

The scenario we described to both the expert panel and, later on, the surveyed users revolves around robotaxis that drive without a driver and can be ordered by potential passengers, for example, via a smartphone app. One vehicle can transport several passengers which not necessarily have to know each other in advance but can get into the taxi one by one. Although the taxis are AVs equipped to handle most driving situations autonomously, in some rare cases, they might be operated remotely by a human, i.e., when they cannot evaluate or resolve the situation on their own. One example could be a situation where a road is blocked by an accident and traffic is diverted for a short time over an area that is usually not accessible (e.g., a one-way street or a sidewalk). In this case, the taxi can be controlled by a tele-pilot via the Internet. The tele-pilot accesses the vehicle's sensors (most notably the cameras) and controls the taxi based on the sensor feed. The remote control can also be used in emergencies, e.g., to navigate the vehicle to the nearest hospital if a passenger needs medical assistance. To detect such emergencies in the vehicle, the taxi would have to be monitored by internal-facing sensors such as cameras or microphones.

After explaining the scenario to the experts, following the NGT method, they were asked to develop ideas for attributes and attribute levels individually and without interaction with the other expert group members. Subsequently, the experts alternately presented their attributes and levels, without discussion at this stage. Afterward, the expert group discussed all presented attributes and levels, thereby identifying additional attributes and clarifying the proposed ideas. In the final step, each expert individually wrote down five attributes with attribute levels and ranked them. We then chose the three highest-ranking attributes of all experts to be used in the CBC. This resulted in the selection of the attributes "possibility of intervention", "pilot", and "interior monitoring" with the attribute levels listed in Table 1. 
Table 1. Attributes and attribute levels

\begin{tabular}{|l|l|l|}
\hline Attributes & Range & Levels \\
\hline Possibility of intervention & 3 & $\begin{array}{l}\text { No possibility of passenger intervention; } \\
\text { Communication channel between the pilot and passengers; } \\
\text { Passengers can override pilot decisions }\end{array}$ \\
\hline Pilot & 3 & $\begin{array}{l}\text { Trained human pilot; } \\
\text { Specialized artificial intelligence (AI); } \\
\text { AI under human supervision }\end{array}$ \\
\hline Interior monitoring & 3 & $\begin{array}{l}\text { Always; } \\
\text { During remote control; } \\
\text { Only after activation by passenger }\end{array}$ \\
\hline Price per kilometer & $\begin{array}{l}2.50 € / \mathrm{km} \text { (similar to taxi); } \\
1.50 € / \mathrm{km} \text { (price between public transport and taxi); } \\
0.5 € / \mathrm{km} \text { (similar to public transport) }\end{array}$ \\
\hline
\end{tabular}

The expert group considered the attribute possibility of intervention the most relevant regarding user acceptance of teleoperable robotaxis. They identified three attribute levels representing varying levels of options to intervene in teleoperation of robotaxis. At the lowest level, the user has no means to intervene in teleoperation. At the middle level, the user can communicate with the remote pilot, and at the highest level, the user can actively intervene and override the pilot's decisions.

The attribute with the second-highest ranking by the expert group relates to the pilot and user trust in the pilot. The expert group identified three levels of automation regarding the remote pilot. The lowest level is a trained human pilot. The highest level with full automation is a specialized artificial intelligence (AI) in the backend, which can access more data and larger computing capacities than the control logic inside the vehicle. The middle level is characterized by a combination of the previous ones so that the AI controls the vehicle while a human pilot monitors it and intervenes when necessary.

The attribute ranked third by the experts concerns interior monitoring of the vehicle, e.g., by cameras or other sensors. The experts identified three levels with the lowest representing an always-on mode of monitoring. In the middle level, interior monitoring is only activated during teleoperation. In the highest level, interior monitoring is switched off by default but can be activated by the user.

The results of our expert panel are consistent with current research, which shows that safety is a crucial but controversial aspect influencing the willingness to use AVs. Some studies show consumers' full confidence in fully automated vehicles (e.g., [33]). At the same time, participants in other surveys prefer partial automation not to lose control entirely and thus be dependent on the technology (e.g., [23, 40]).

Besides the expert-chosen attributes, we included price as a fourth attribute, as it plays a particularly important role when purchasing or renting emerging, innovative technologies [22]. More specifically, in shared autonomous taxis, travel costs are among the most relevant factors predicting adoption [24]. For the current range of on-demand as well as car-sharing options, price models are often usage-based, so we also included the price per kilometer as an attribute in our study. The average kilometer price for a taxi in Germany, where the study was conducted, is between $2.50 €$ and $3 €$ for shorter distances. For local public transport, the value is approximately $0.5 €$ per kilometer. Moreover, previous autonomous vehicle literature assumes that the operating costs of passenger cars will remain unchanged, whereby robotaxis could become the cheapest transport option thanks to substantial savings in labor costs so that the price per kilometer will be essentially lower than that of taxis in use today [4]. We have therefore included $0.5 € / \mathrm{km}$ as a price level similar to public transport, $1.50 € / \mathrm{km}$ as an intermediate price level between public transportation and taxi, and $2.50 € / \mathrm{km}$, which corresponds to the taxi costs

In alignment with Street and Burgess [42], we use a Doptimal ( $3 \times 3 \times 3 \times 3)$ fractional factorial design with 14 choice sets of which 12 choice sets are used for the estimation and the remaining two choice sets serve as holdouts to assess the predictive validity. Each choice set consists of three autonomous, teleoperable taxis, from which the participants must choose their preferred option. Figure 1 provides a translated illustration of such a choice set. 


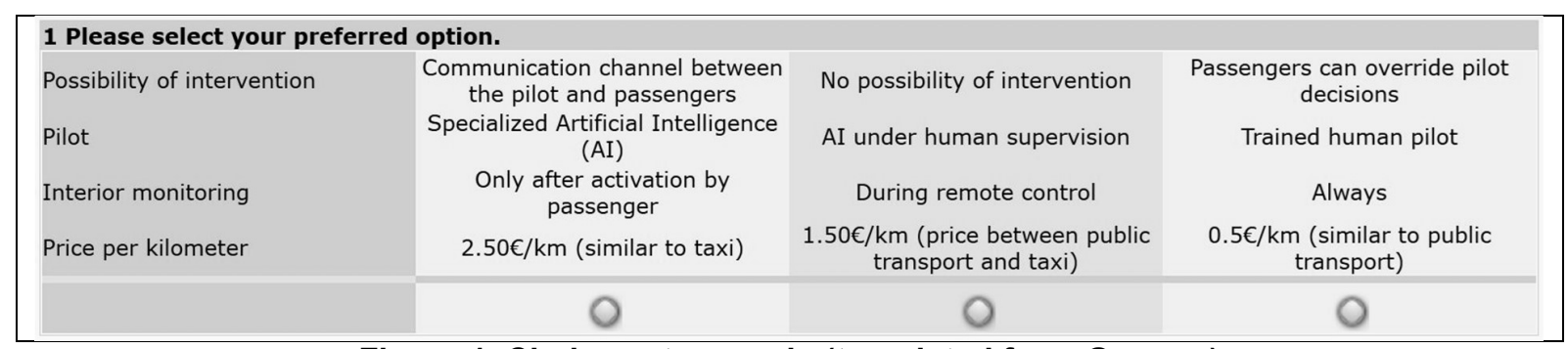

Figure 1. Choice set example (translated from German)

The survey began with a short introduction to the topic of robotaxis and teleoperation and a presentation of all attributes and their attribute levels. Afterward, we collected demographic and psychographic data of the participants before finally proceeding to the choice-based conjoint part where the survey participants chose their preferred teleoperable robotaxi based on the above-described attributes and we thus recorded the user preferences. We implemented our study in DISE [38].

\subsection{Data Collection}

We conducted our study in November 2018 and hired a market research firm to receive a representative sample. We received 546 completed questionnaires, which we used for our analysis. For 491 participants, we have information on age and gender, see Table 2. Of those participants, $45.24 \%$ are female and $44.69 \%$ male. We observed a relatively even age distribution, with slightly more respondents in the age groups 18 27, 48-57 and 58-67.

\subsection{Model Assessment}

For the estimation, we follow the utility-theoretic approach [44], which assumes that an individual $h$ chooses alternative $i$ that offers the highest utility $u_{h, i}$. The utility consists of a deterministic part and a stochastic part: $u_{h, i}=v_{h, i}+\varepsilon_{h, i}$. The stochastic part $\varepsilon_{h, i}$ contains unobservable behavior for which we assume an extreme value distribution that has a similar functional form as the normal distribution. The deterministic part $v_{h, i}$ contains observable information, such as the attributes and levels shown in the selected sets. We use an additive model for the utility of a product alternative, i.e., $v_{h, i}=\beta_{h} \cdot X_{i}$, where $\beta_{h}$ is a vector of the preferences of respondent $h$ for all attributes and vector $X_{i}$ indicates the attribute levels of each attribute in the product $i[16]$.
Table 2. Demographics of respondents

\begin{tabular}{|l|l|}
\hline Gender & Age \\
\hline Female (45.24\%) & $18-27(17.22 \%)$ \\
\hline Male (44.69\%) & $28-37(10.99 \%)$ \\
\hline Not indicated (10.07\%) & $38-47(9.71 \%)$ \\
\hline & $48-57(17.95 \%)$ \\
\hline & $58-67(23.08 \%)$ \\
\hline & $68+(10.99 \%)$ \\
\hline & Not indicated $(10.07 \%)$ \\
\hline
\end{tabular}

For each choice set $a$, we estimate the probability that respondent $h$ selects an option $i$ as the preferred alternative from the set of alternatives $I$ :

$$
\operatorname{Pr}_{h, a}(i)=\frac{\exp \left(\mathrm{v}_{\mathrm{h}, \mathrm{i}}\right)}{\sum_{\mathrm{i}, \in \mathrm{I}} \exp \left(\mathrm{v}_{\mathrm{h}, \mathrm{i}}\right)}
$$

For the estimation of the individual-level parameters from the choice-based conjoint analysis data, we employ Hierarchical Bayes (HB). Accordingly, we estimate the parameter values based on the respondents' choices and deduce the importance weights for the attributes (see Table 3 ).

The signs and magnitudes of the parameter values are all consistent and reasonable, providing high face validity. For example, a higher price per kilometer results in a lower utility. To further assess the validity of our results, we consider the proportion of correctly predicted choices based on the first-choice-model and observe an internal validity of $83 \%$. In addition, we use two holdout choice sets to estimate external validity and obtain a value of $68 \%$. The goodness-offit-measures thus indicate reasonably good results.

The aggregated parameter values (see Table 3) show that our participants strongly prefer a communication channel between the pilot and passengers as a means of intervention (0.63), followed by the possibility of overriding pilot (0.49). Furthermore, our participants clearly prefer the trained human pilot (0.58) and interior monitoring only after activation by the passenger $(0.22)$. 
Table 3. Parameter estimates and average importance weights

\begin{tabular}{|c|c|c|c|c|c|}
\hline Attributes & Levels & $\begin{array}{l}\text { Aggregated } \\
\text { Parameter } \\
\text { Values } \\
\text { (Std. Dev.) }\end{array}$ & $\begin{array}{l}\text { Minimal } \\
\text { Individual } \\
\text { Parameter } \\
\text { Value }\end{array}$ & $\begin{array}{l}\text { Maximal } \\
\text { Individual } \\
\text { Parameter } \\
\text { Value }\end{array}$ & $\begin{array}{l}\text { Average } \\
\text { Importance } \\
\text { Weights (Std. } \\
\text { Dev.) }\end{array}$ \\
\hline \multirow{3}{*}{$\begin{array}{l}\text { Possibility of } \\
\text { intervention }\end{array}$} & $\begin{array}{l}\text { No possibility of } \\
\text { passenger } \\
\text { intervention }\end{array}$ & $-1.12(0.92)$ & -2.93 & 2.27 & \multirow{3}{*}{$\begin{array}{l}30.82 \% \\
(18.52 \%)\end{array}$} \\
\hline & $\begin{array}{l}\text { Communication } \\
\text { channel between the } \\
\text { pilot and passengers }\end{array}$ & $0.63(0.56)$ & -0.89 & 2.20 & \\
\hline & $\begin{array}{l}\text { Passengers can } \\
\text { override pilot } \\
\text { decisions }\end{array}$ & $0.49(0.74)$ & -1.47 & 2.39 & \\
\hline \multirow{3}{*}{ Pilot } & Trained human pilot & $0.57(0.76)$ & -1.11 & 2.37 & \multirow{3}{*}{$\begin{array}{l}22.34 \% \\
(15.73 \%)\end{array}$} \\
\hline & $\begin{array}{l}\text { Specialized artificial } \\
\text { intelligence }(\mathrm{AI})\end{array}$ & $-0.64(0.55)$ & -1.75 & 0.75 & \\
\hline & $\begin{array}{l}\text { AI under human } \\
\text { supervision }\end{array}$ & $0.07(0.40)$ & -0.91 & 1.72 & \\
\hline \multirow{3}{*}{ Interior monitoring } & Always & $-0.18(0.26)$ & -0.90 & 0.61 & \multirow{3}{*}{$\begin{array}{l}12.03 \% \\
(11.54 \%)\end{array}$} \\
\hline & During remote control & $-0.04(0.33)$ & -1.03 & 1.13 & \\
\hline & $\begin{array}{l}\text { Only after activation } \\
\text { by passenger }\end{array}$ & $0.22(0.50)$ & -1.12 & 1.87 & \\
\hline Price & Price per kilometer & $-3.59(7.23)$ & -34.11 & -0.09 & $\begin{array}{l}34.81 \% \\
(26.34 \%)\end{array}$ \\
\hline
\end{tabular}

Looking at the average importance weights in Table 3, we observe that price is the most crucial attribute in the purchase decision (34.81\%). At the same time, it also has the largest standard deviation $(26.34 \%)$, indicating heterogeneous preferences. We obtain similar results for the second most important attribute possibility of intervention (30.82\% average importance weight, $18.52 \%$ standard deviation).

In the next step, we take a closer look at the influence of taxi design on the willingness to pay. Therefore, we estimate the equalization prices (see Table 4) between the least and the most preferred attribute levels based on the average parameter values. To maintain a communication channel between the pilot and the passengers instead of no possibility of passenger intervention, our respondents are, on average, willing to pay $2.76 €$ per $\mathrm{km}$. Furthermore, our participants are willing to pay an additional $1.54 €$ per $\mathrm{km}$ for a trained human pilot instead of a specialized AI. However, the willingness to pay for control over interior monitoring is rather low $(0.12 €$ per $\mathrm{km}$ for interior monitoring only after activation by passenger compared to always).

Both the high standard deviations of the importance weights and the considerable differences between minimum and maximum values of the individual parameter values show that the participants' preferences are highly heterogeneous. Therefore, we conduct a cluster analysis to identify user segments for understanding the market on a segment-specific basis. To find the optimum number of segments and obtain sufficient stability, we apply the "Elbow criterion" [2]. Observing a jump in heterogeneity development at that value, we decide to use five clusters to obtain a manageable number of segments while keeping them as homogeneous as possible.

Table 5 presents the results of the cluster analysis with the average net income based on the participants who provided information on their income.

Cluster 1 ("Parents"), as the second-largest cluster, perceives the possibility of intervention as the most important attribute and, accordingly, shows a high willingness to pay. This segment has strong preferences for both attribute levels, that passengers can override pilot decisions and a communication channel between pilot and passengers. The participants belonging to this segment have a high net income, are married, and have children. Therefore, the possibility of intervention might be a safety factor for them and their families. 
Table 4. Equalization prices

\begin{tabular}{|l|l|}
\hline & $\begin{array}{l}\text { Equalization } \\
\text { price }\end{array}$ \\
\hline $\begin{array}{l}\text { Possibility of intervention } \\
\text { (Communication channel between } \\
\text { the pilot and passengers vs. } \\
\text { No possibility of intervention) }\end{array}$ & $2.76 €$ \\
\hline $\begin{array}{l}\text { Pilot } \\
\text { (Trained human pilot vs. } \\
\text { Specialized artificial intelligence) }\end{array}$ & $1.54 €$ \\
\hline $\begin{array}{l}\text { Interior monitoring } \\
\text { (Only after activation by passenger } \\
\text { vs. Always) }\end{array}$ & $0.12 €$ \\
\hline
\end{tabular}

Cluster 2 ("Metropolitans") is the smallest cluster consisting of people predominantly living in large or medium-sized cities and who also have the lowest net income. The majority of respondents in this cluster $(62.85 \%)$ do not have a driving license. Besides, public transport is their preferred and most used mode of transport. They show slight preferences for the possibility of intervention and interior monitoring, but no clear positioning in terms of importance weights.

Cluster 3 ("Traditionalists") shows strong preferences for the attribute pilot, with a human pilot strongly preferred. The other attributes are rather unimportant. This segment consists of a relatively rural population with medium income.

Cluster 4 ("Price Sensitives") contains pricesensitive individuals with a strong preference for low prices, while the other attributes are rather unimportant. Correspondingly, they also show low compensatory prices. It is the third-largest cluster with mixed demographics and middle income.

Cluster 5 ("Urbanites"), as the largest cluster, perceives the possibility of intervention, pilot, and interior monitoring as more important than price. It consists mainly of singles or individuals in childless partnerships with relatively low income living in the suburbs or a big city.

In addition, we performed an analysis of variance (One-way ANOVA) to observe possible influences of age, gender, marital status, number of children, employment, net income, means of transport, place of residence, and driving license on our attributes.

We observe a strong statistically significant influence of gender on the possibility of intervention $(\mathrm{p}<0.001)$ and price per $\mathrm{km}(\mathrm{p}<0.05)$ (see Table 6 , Appendix). While female participants perceive the possibility of intervention as more important than male participants, male participants have stronger preferences for the attribute price. Although men in earlier studies were significantly more likely to choose the full degree of automation for the safety function [40] and showed a higher willingness to use fully automated vehicles (e.g., [17]), while women were more afraid of automated cars than men [17], we observe very similar parameter values for both genders. However, with an average parameter value of 3.86 , the male participants in our study are far more price-sensitive than female participants with an average value of 2.93 .

Moreover, age has a significant influence on the possibility of intervention $(\mathrm{p}<0.1)$ (see Table 7, Appendix). The middle age group (38-47) with the lowest significance weight of $26.47 \%$, and the oldest participants $(68+)$ with the highest significance weight $(33.18 \%)$, are particularly striking.

We also find significant influence $(\mathrm{p}<0.1)$ of net income on the attribute pilot (see Table 8, Appendix). Participants with higher earning $(3000 €+)$ have a lower significance weight for the attribute "pilot", while this attribute is more important for participants with no own, low, or middle income. The other sociodemographic characteristics have no significant effect on the level of importance weights.

\section{Discussion of the Results}

In addition to our investigation's novel subject, we avoid several pitfalls associated with earlier work on AVs. Previous studies often suffer from a focus on respondents who currently use a car, which can lead to biased results [31]. Instead, we aim at a representative sample of the German population as a whole and reach a broad sample with demographics akin to the general population. Our study object, teleoperable robotaxis, is also closer to respondents' experiences, e.g., with remote controlled drones and toy cars, reducing another source of bias in previous studies [31]. With our study, we also follow the call of Stoiber et al. [41] and investigate the preferences for autonomous taxis to address the characteristics of different social segments as well as possible socio-demographic factors.

We observe price as the most important influencing factor for teleoperable robotaxis, which is in line with Papadima et al. [33], who found ticket price as the crucial attribute for the use of driverless busses. However, the price only accounts for about one third of the evaluation. Interestingly, the possibility of intervention, communicating, or even overriding the remote pilot's decisions follows second. Hence, it is significantly more relevant than the remote pilot's qualification and even whether the remote pilot is a human or an AI. 
Table 5. Cluster analysis

\begin{tabular}{|c|c|c|c|c|c|}
\hline Cluster (cluster size) & $\begin{array}{l}\text { Parents } \\
\text { (139) }\end{array}$ & $\begin{array}{l}\text { Metropolitans } \\
(70)\end{array}$ & $\begin{array}{l}\text { Traditionalists } \\
(80)\end{array}$ & $\begin{array}{l}\text { Price } \\
\text { Sensitives } \\
(113)\end{array}$ & $\begin{array}{l}\text { Urbanites } \\
(144)\end{array}$ \\
\hline IW Possibility of intervention & 0.6681 & 0.2076 & -0.2820 & -1.0943 & 0.2695 \\
\hline IW Pilot & -0.3092 & 0.0523 & 1.3701 & -0.9164 & 0.2310 \\
\hline IW Interior monitoring & 0.3967 & 0.1770 & -0.4278 & -0.6724 & 0.2964 \\
\hline IW Price & -0.4591 & -0.2548 & -0.4324 & 1.6117 & -0.4574 \\
\hline Big city & $24.46 \%$ & $54.29 \%$ & $27.50 \%$ & $35.40 \%$ & $41.67 \%$ \\
\hline Medium sized city & $44.60 \%$ & $30.00 \%$ & $38.75 \%$ & $37.17 \%$ & $40.97 \%$ \\
\hline Rural area & $30.94 \%$ & $15.71 \%$ & $33.75 \%$ & $27.43 \%$ & $17.36 \%$ \\
\hline Average net income & 7.56 & 3.77 & 5.6 & 6.03 & 4.39 \\
\hline EP Possibility of intervention & $3.60 €$ & $3.19 €$ & $2.62 €$ & $0.26 €$ & $3.78 €$ \\
\hline EP Pilot & $1.09 €$ & $1.11 €$ & $3.59 €$ & $0.20 €$ & $2.10 €$ \\
\hline EP Interior monitoring & $0.31 €$ & $-0.10 €$ & $-0.20 €$ & $0.07 €$ & $0.27 €$ \\
\hline
\end{tabular}

Interior monitoring of the vehicle seems to be a relatively minor concern, but, in general, has a negative impact, unless it is - once again - triggered by passenger interaction, in which case the system receives a positive valuation, further emphasizing the significance of user agency in the AV scenario we investigated.

\subsection{Implications for Theory and Practice}

We can derive implications for both theory and practice from the empirical results of our study.

Robotaxis are currently being deployed by many companies (cf. [14]). Whether their service will be adopted by customers will also depend on customers' trust, risk, and control perceptions. Fraedrich et al. [12] find that, while a small majority of respondents can image using on-demand AVs (i.e. robotaxis), $45 \%$ of the sample were skeptical. However, the authors anticipate "the biggest declines in usage [...] for the taxi" and expect "using a taxi $[\ldots]$ to become less common with the availability of [AVs]" [12].

Teleoperation can smoothen the transition from highly automated (SAE Level 4) to SAE Level 5 robotaxis that can handle any situations without human intervention. However, our findings show an urge to intervene and suggest emphasizing mechanisms for facilitating user control in teleoperation. Emphasizing means of control for passengers, communicating with and potentially overruling decisions of the tele-pilot, is a new impulse, that may motivate a redesign of current driverless pods, which are known to have no steering wheel [21] and often have an emergency stop as the single point of intervention for passengers [35]. This finding aligns with theory from other areas of IS, indicating that perceived control impacts perceived risk, and thus affect intention to use [5]. Our results also align with previous research showing that user interfaces in AVs enabling advanced interaction build trust, a sense of control, and enjoyment [25].

Hence, our findings suggest that hybrid solutions including vehicle-side AI and human-controlled teleoperation and possibilities of intervention regarding teleoperation might be promising approaches towards building trust in AVs. Such systems may also pave the way towards the acceptance of fully automated vehicles, in particular given the vastly heterogeneous preferences regarding AI-controlled systems.

\subsection{Limitations and Future Research}

For new technologies, user preferences are usually not yet strongly developed and can change over time. Thus, the development of user acceptance regarding autonomous taxis should be surveyed over time. First, studies show that perceived risk may fall significantly as users get familiar with autonomous vehicles, which may impact the desire for intervention we observed.

In any case, we encourage further research regarding the role of various forms of interaction and intervention in the acceptance of AVs, as we could only cover one specific use case with users from Germany. As previous research indicates the AV space to be quite heterogeneous in user perception, we encourage broader research in this space.

\section{Conclusion}

Our study contributes to a better understanding of user preferences for autonomous and teleoperable taxis. This topic is highly relevant, as such systems might 
smoothen the transition from partly automated to fully automated vehicles. In particular, teleoperation is a promising approach to utilizing highly automated vehicles (SAE Level 4) for taxi services without burdening passengers with the tasks of handling the vehicle when it reaches the limits of its functionality.

The consideration of different attributes in interaction with user control in on-demand AVs is new and unique, but essential for the users as previous literature shows (e.g., $[11,25])$. We base the selection of attributes on interviews with experts who have identified different control aspects as a key issue for this mobility scenario.

With our $\mathrm{CBC}$, we understand driving factors for adopting teleoperable robotaxis and obtain insights into importance weights and purchase probabilities, leading to manifold insights. For example, price is the most important attribute, closely followed by the possibility of intervention. This indicates that, in addition to optimal pricing, manufacturers can also increase the acceptance of this essential technology by offering users interfaces for intervention options.

For further analysis, we conduct a cluster analysis and look at different customer segments. For example, we observe that the second-largest segment perceives the possibility of intervention as the most important attribute and also shows a high willingness to pay for it. Using an analysis of variance, we also analyze possible moderation effects and discover, for example, that female participants have stronger preferences for the possibility of intervention than male ones, while male participants find the price more important.

\section{Acknowledgement}

The work on the paper was partly funded by the German Research Foundation (DFG) within the Collaborative Research Center (CRC) 1053 - MAKI, the German Federal Ministry for Economic Affairs and Energy in the projects ENTOURAGE (Smart Services Worlds Innovation Competition) and ForeSight (Artificial Intelligence Innovation Competition).

\section{References}

[1] H. Abraham, C. Lee, S. Brady, C. Fitzgerald, B. Mehler, B. Reimer, and J. Coughlin, "Autonomous vehicles, trust, and driving alternatives: A survey of consumer preferences", Massachusetts Institute of Technology, Age Lab, Cambridge, 2016.

[2] Backhaus, K., B. Erichson, W. Plinke, and R. Weiber, Multivariate Analysemethoden - Eine anwendungsorientierte Einführung, Springer, Berlin, 2016.
[3] F. Becker, and K.W. Axhausen, "Literature Review on Surveys Investigating the Acceptance of Automated Vehicles", Transportation, 44(6), 2017, pp. 1293-1306.

[4] P.M. Bösch, F. Becker, H. Becker, and K.W. Axhausen, "Cost-Based Analysis of Autonomous Mobility Services”, Transport Policy, 64, 2018, pp. 76-91.

[5] L. Brandimarte, A. Acquisti, and G. Loewenstein, "Misplaced Confidences: Privacy and the Control Paradox", Social Psychological and Personality Science, 4(3), 2013, pp. 340-347.

[6] J.K. Choi, and Y.G. Ji, "Investigating the Importance of Trust on Adopting an Autonomous Vehicle", International Journal of Human-Computer Interaction, 31(10), 2015, pp. 692-702.

[7] R.A. Daziano, M. Sarrias, and B. Leard, "Are Consumers Willing to Pay to Let Cars Drive for Them? Analyzing Response to Autonomous Vehicles", Transportation Research Part C: Emerging Technologies, 78, 2017, pp. 150-164.

[8] D.M. DeJoy, "The Optimism Bias and Traffic Accident Risk Perception", Accident Analysis and Prevention, 21(4), 1989, pp. 333-340.

[9] D.A.L. Delbecq, and A. Van de Ven, "Nominal Group Techniques for Involving Clients and Resource Experts in Program Planning", Academy of Management Proceedings, 1970, pp. 208-227.

[10] C.P.H. Ernst, and P. Reinelt, "Autonomous Car Acceptance: Safety vs. Personal Driving Enjoyment", Americas Conference on Information Systems, 2017.

[11] M. Erskine, and S. Brooks, "Attitude and Behavioral Intentions Regarding Autonomous Automobiles: Effects of Emotional Response and Locus of Control", Americas Conference on Information Systems, 2019.

[12] Fraedrich, E., R. Cyganski, I. Wolf, and B. Lenz, User perspectives on autonomous driving: A use-case-driven study in Germany, 2016.

[13] C.J. Haboucha, R. Ishaq, and Y. Shiftan, "User Preferences Regarding Autonomous Vehicles", Transportation Research Part C: Emerging Technologies, 78, 2017, pp. 37-49.

[14] A.J. Hawkins, "Mercedes-Benz and Bosch are testing self-driving taxis in San Jose", 2019. https://www.theverge.com/2019/12/9/21002911/merced es-benz-bosch-self-driving-taxi-car-sclass-san-joseautonomous, Accessed on 13.10.2020.

[15] D. Hein, P.A. Rauschnabel, J. He, L. Richter, and B.S Ivens, "What Drives the Adoption of Autonomous Cars?", International Conference on Information Systems, 2018.

[16] O. Hinz, C. Schlereth, and W. Zhou, "Fostering the adoption of electric vehicles by providing complementary mobility services: a two-step approach using Best-Worst Scaling and Dual Response", Journal of Business Economics, 85(8), 2015, pp. 921-951.

[17] C. Hohenberger, M. Spörrle, and I.M. Welpe, "How And Why Do Men and Women Differ in Their Willingness to Use Automated Cars? The Influence of Emotions Across Different Age Groups", Transportation Research Part A: Policy and Practice, 94, 2016, pp. 374-385.

[18] A. Hosseini, and M. Lienkamp, "Predictive Safety Based on Track-Before-Detect for Teleoperated Driving 
Through Communication Time Delay", IEEE Intelligent Vehicles Symposium, 2016, pp. 165-172.

[19] D. Howard, and D. Dai, "Public Perceptions of Selfdriving Cars: The Case of Berkeley, California", 93rdAnnual Meeting of the Transportation Research Board, 2014.

[20] L. Hulse, H. Xie, and E.R. Galea, "Perceptions of autonomous vehicles: Relationships with road users, risk, gender and age", Safety Science, 102, 2018, pp. 1-13.

[21] C. Jee, "GM's Cruise Has Unveiled a Self-Driving Car With No Steering Wheel", 2020. https://www.technologyreview.com/2020/01/22/276146/ gms-cruise-has-unveiled-a-self-driving-car-with-nosteering-wheel/, Accessed on 13.07.2020.

[22] P.K. Kapur, O. Singh, U. Chanda, and M. Basirzadeh, "Determining Adoption Pattern With Pricing Using TwoDimensional Innovation Diffusion Model", Journal of High Technology Management Research, 21(2), 2010, pp. 136-146.

[23] M. König, and L. Neumayr, "Users' Resistance Towards Radical Innovations: The Case of the Self-Driving Car", Transportation Research Part F: Traffic Psychology and Behaviour, 44, 2017, pp. 42-52.

[24] R. Krueger, T.H. Rashidi, and J.M. Rose, "Preferences for Shared Autonomous Vehicles", Transportation Research Part C: Emerging Technologies, 69, 2016, pp. 343-355.

[25] D.R. Large, K. Harrington, G. Burnett, and J. Luton, "To Please in a Pod: Employing an Anthropomorphic AgentInterlocutor to Enhance Trust and User Experience in an Autonomous, Self-Driving Vehicle", International Conference on Automotive User Interfaces and Interactive Vehicular Applications, 2019

[26] J.G. Lee, K.J. Kim, S. Lee, and D.-H. Shin, "Can Autonomous Vehicles Be Safe and Trustworthy? Effects of Appearance and Autonomy of Unmanned Driving Systems", International Journal of Human-Computer Interaction, 31(10), 2015, pp. 682-691.

[27] P. Lenk, W.S. Desarbo, P.E. Green, and M.R. Young, "Hierarchical Bayes Conjoint Analysis - Recovery of Partworth Heterogeneity from Reduced Experimental Designs", Marketing Science, 15(2), 1996, pp. 173-191.

[28] C. Linehan, Murphy, G., Hicks, K., Gerling, K., Morrissey, K., "Handing over the keys: a qualitative study of the experience of automation in driving", International Journal of Human-Computer Interaction, 35(18), 2019.

[29] K. Maeng, and Y. Cho, "A Study on Consumer Preference of Autonomous Vehicles' Connectivity: The Case of South Korea", ToKnowPress, 2018.

[30] K. Mühl, C. Strauch, C. Grabmaier, S. Reithinger, A. Huckauf, and M. Baumann, "Get Ready for Being Chauffeured: Passenger's Preferences and Trust While Being Driven by Human and Automation", Human factors, 2019.

[31] S. Nordhoff, B. Van Arem, and R. Happee, "Conceptual Model to Explain, Predict, and Improve User Acceptance of Driverless Podlike Vehicles", Transportation Research Record, 2602(1), 2016, pp. 60-67.

[32] S. Nordhoff, J. De Winter, M. Kyriakidis, B. Van Arem, and R. Happee, "Acceptance of Driverless Vehicles:
Results from a Large Cross-National Questionnaire Study", Journal of Advanced Transportation, 2018.

[33] G. Papadima, E. Genitsaris, I. Karagiotas, A. Naniopoulos, and D. Nalmpantis, "Investigation of Acceptance of Driverless Buses in the City of Trikala and Optimization of the Service Using Conjoint Analysis", Utilities Policy, 62, 2020, pp. 100994.

[34] Raghavarao, D., J.B. Wiley, and P. Chitturi, ChoiceBased Conjoint Analysis: Models and Designs, 2010.

[35] Reschka, A., "Safety Concept for Autonomous Vehicles", In M. Maurer, C. Gerdes, B. Lenz and H. Winner, eds., Autonomous Driving: Technical, Legal and Social Aspects. Spinger-Verlag Berlin Heidelberg, 2016.

[36] C. Rödel, S. Stadler, A. Meschtscherjakov, and M. Tscheligi, "Towards autonomous cars: The effect of autonomy levels on acceptance and user experience", International Conference on Automotive User Interfaces and Interactive Vehicular Applications, (2014).

[37] D. Ruyter, "Focus Versus Nominal Group Interviews: A Comparative Analysis", Marketing Intelligence \& Planning, 14(6), 1996, pp. 44-50.

[38] C. Schlereth, and B. Skiera, "DISE: Dynamic Intelligent Survey Engine", In Quantitative Marketing and Marketing Management. 2012, 225-243.

[39] Society of Automotive Engineers, "Taxonomy and Definitions for Terms Related to On-Road Motor Vehicle Automated Driving Systems", 2014. https://saemobilus.sae.org/content/33016 201401, Accessed on 29.09.2020.

[40] M. Souka, D. Böger, R. Decker, C. Stummer, and A. Wiemann, "Is More Automation Always Better? An Empirical Study of Customers' Willingness to Use Autonomous Vehicle Functions", International Journal of Automotive Technology and Management, 20(1), 2020, pp. 1-24.

[41] T. Stoiber, I. Schubert, R. Hoerler, and P. Burger, "Will Consumers Prefer Shared and Pooled-Use Autonomous Vehicles? A Stated Choice Experiment with Swiss Households", Transportation Research Part D: Transport and Environment, 71, 2019, pp. 265-282.

[42] Street, D.J., and L. Burgess, The Construction of Optimal Stated Choice Experiments: Theory and Methods, 2007.

[43] S. Szymkowski, "Audi hangs up hopes for Level 3 partial automation system", 2020 https://www.cnet.com/roadshow/news/audi-a8-level-3automation-traffic-jam-pilot-system/, Accessed on 29.09.2020

[44] L.L. Thurstone, "A Law of Comparative Judgment", Psychological Review, 34(4), 1927, pp. 273.

[45] P. Wintersberger, and A. Riener, "Trust in Technology as a Safety Aspect in Highly Automated Driving", i-com, 15(3), 2016

[46] Z. Xu, K. Zhang, H. Min, Z. Wang, X. Zhao, and P. Liu, "What Drives People to Accept Automated Vehicles? Findings From a Field Experiment", Transportation Research Part C: Emerging Technologies, 95, 2018, pp. 320-334.

[47] J.C. Zoellick, A. Kuhlmey, L. Schenk, D. Schindel, and S. Blüher, "Assessing acceptance of electric automated vehicles after exposure in a realistic traffic environment", PloS one, 14(5), 2019 